\title{
Make scientific conferences count
}

\author{
Travelling to a conference can be financially and environmentally costly, researchers should take careful decisions \\ on which meetings to attend and put in place a strategy to extract as much benefit from the event as possible.
}

T he peak of the microbiology conference season is almost upon us, but why in the age of instant messaging, video calling, webinars, e-mail and social media is travelling still necessary? Considering this question is important given that scientists (who are perhaps likely to be more environmentally aware than average) may invite accusations of hypocrisy by enlarging their carbon footprint undertaking expensive journeys to attend a meeting halfway around the world. So why do we still do it? Certainly a conference gets us out of the laboratory and away from our desk/bench, providing a refreshing change of scenery. Furthermore, conferences are often held in interesting or exotic places that are desirable to visit. Yet, more than just being an excuse for a jolly, conferences provide a chance to interact in person with others in the field, talk with delegates about data presented, share gossip about the field, discuss new technologies, new papers and funding opportunities, and much more. Yes, all of this communication could be electronic, but much of the quality of such interactions is lost online. With character limits, buffering and response delays, spontaneity, personality and nuance can be absent as compared to a face-to-face interaction between a group of researchers, or between a speaker and a live audience. The importance of conference attendance has thus not waned with technological advances. Rather, being at an event in person enables community building, and helps us to process our own thinking and understanding of the 'geography' of the field, which is essential when it comes to strategic planning for future experiments or career choices.

If attendance at conferences is still necessary then, to minimize any guilt arising from the fuel and finances burned to get there, travelling researchers should aim to squeeze every last drop of opportunity from an event. The first task is to determine the key reasons why attending a conference is being considered in the first place. This will vary from individual to individual, the specifics of their current role and career stage, as well as future plans. Early career researchers may be using a conference to obtain a broader overview of the research being carried out in their field or sub-field, and they may also be looking to make connections that will help them plot a future step in their career. Those more established may be looking to recruit lab members or entice potential faculty to joining their institution. All are likely to be looking for an opportunity to share their work, whether as a speaker or a poster presenter, and hear about the latest work from colleagues in the field.

The next step is to look for a suitable conference in the calendar that matches with the aims to be realized. Is it a large or small meeting? Each comes with benefits and drawbacks; large meetings can often cover more ground scientifically but tend to be quite impersonal, whereas smaller meetings, while more limited in scope, will give a deeper view and are likely to provide plenty of opportunity for interaction with others. Are the key people in the field going to be attending? Does the conference provide a diverse line-up of speakers that is inclusive of views and topics from all different corners of the field and that is more likely to create a thought-provoking meeting? Is there an opportunity for submitting an abstract for a poster or to speak? How much will the trip cost and are bursaries available? Are there any other professional opportunities that can be added on to the trip, such as visiting local institutions and meeting collaborators? Equally important to ask is whether this is a trip that is likely to be repeated again, and if not, is it possible to take some personal time to experience the local culture? Does the conference fit with a well-balanced work and home life and would it be possible take a partner/family; are there childcare facilities provided or interesting activities to keep loved ones occupied in the vicinity?

When a conference has been settled on, there is still much more that can be done to squeeze every last drop of as-yet unrealized opportunity out of the meeting. An attendee could look at who is speaking to identify anyone known or with whom making contact would be useful. This can be intimidating, especially for junior researchers, but reaching out in advance to request a short meeting at the conference is more effective than just hoping to bump into someone. Although there will be exceptions in every field of research, the overwhelming majority of researchers are nice people, who enjoy making new contacts. Go through the conference programme and work out which talks have to be seen, and which would be nice to see. If the conference has parallel sessions, check the map of the venue to make sure that moving between two talks is realistic if jumping between sessions.

Once at the conference, make sure to take notes. There is a lot going on, even at smaller meetings, and the days will be long. Without notes, some of the useful information that comes up will be forgotten. During talks and interactions with other delegates, join in the discussion and make sure to ask questions. This can be intimidating, especially in a packed lecture hall, but if you have a particular question it will likely be shared by others in the audience, so raising it can help to make a speaker's data and thinking clearer for all. Alternatively, saving the question to ask of a speaker at the end of a session can be a great way to make contact. Check if the meeting is concurrently happening on Twitter, follow the conference hashtag and use it to make acquaintances with people offline. Learn people's names, repeat and remember, exchange contact details, Twitter handles and business cards. Social gatherings can be a good opportunity to make new contacts and cement existing ones, but keep things professional, behave responsibly around others; drink socially but not excessively, dance if the occasion calls for it, but not if it doesn't. Take the opportunity to sleep well; attendees get more out of each day at a meeting if they are well-rested each night. When the formal conference ends, for the contacts made to be solidified, it is good practice to follow up with a quick message when back in the lab. Finally, edit and order any notes that you have taken while they are fresh in your mind, share them with colleagues in the lab that did not attend the meeting and file them in a way that you will be able to find them easily.

Scientific conferences can be pivotal events in a research career; being proactive and adopting a strategy when selecting and attending will help to make the most of a meeting.

Published online: 24 April 2018 https://doi.org/10.1038/s41564-018-0158-y 